\title{
Realisation of ultra-low loss photonic crystal slab waveguide devices
}

\author{
M.D.B. Charlton ${ }^{\mathrm{a}, \mathrm{c}, *}$, M.E. Zoorob ${ }^{\mathrm{c}}$, M.C. Netti ${ }^{\mathrm{c}}$, N. Perney ${ }^{\mathrm{c}}$, G.J. Parker ${ }^{\mathrm{a}, \mathrm{c}}$, \\ P. Ayliffe ${ }^{c}$, J.J. Baumberg ${ }^{a, b, c}$ \\ ${ }^{\mathrm{a}}$ School of Electronics and Computer Science, University of Southampton, Highfield, Southampton SO17 1BJ, UK \\ ${ }^{\mathrm{b}}$ School of Physics and Astronomy, University of Southampton, Southampton SO17 1BJ, UK \\ ${ }^{\mathrm{c}}$ Mesophotonics Ltd, 2 Venture Rd, Chilworth Science Park, Southampton SO16 7NP, UK
}

\begin{abstract}
In this paper we demonstrate low loss transmission both above and below the primary band-gap for a photonic crystal (PC) super-prism device consisting of 600 lattice periods. By modifying the refractive index of the holes, we reduce overall insertion loss to just $4.5 \mathrm{~dB}$ across the entire visible spectrum. We show that the remaining loss is predominantly due to impedance mismatch at the boundaries between patterned and unpatterned slab waveguide regions. Experimental loss measurements compare well with finite difference time domain simulations.
\end{abstract}

(C) 2005 Elsevier Ltd. All rights reserved.

Keywords: Photonic crystal; Waveguide; Loss

\section{Introduction}

Loss is a major factor limiting photonic crystals (PCs) from competing in key application areas in integrated optics. The key loss mechanism in waveguiding PCs is upscattering from the holes. Applications for PCs can be generalised into two categories.

The first class of applications include 'line defect' [1] and 'CROW' waveguides [2] which make use of the reflective properties of photonic band gaps (PBGs) to obtain transmission along chains of defect sites at wavelengths lying within the PBG of the surrounding lattice. In these applications light is predominantly localised to high refractive index defect regions confined within the surrounding PC lattice. A small proportion of the mode energy is localised as an exponentially decaying evanescent field within a few rows of holes adjacent to the line defect. The small proportion of this field which intersects the spatial regions of the holes is subject to loss.

\footnotetext{
* Corresponding author. Address: School of Electronics and Computer Science, University of Southampton, Room 2043, Mountbatten Building, Highfield, Southampton SO17 1BJ, UK. Tel.: +44 01703 593737; fax: + 4401703593029.

E-mail address: mdc1@soton.ac.uk (M.D.B. Charlton).
}

0026-2692/\$ - see front matter (C) 2005 Elsevier Ltd. All rights reserved. doi:10.1016/j.mejo.2005.02.105
The second class of applications include devices such as PC superprisms [3,4] and optical time-delay elements [5,6] which make use of the dispersive properties of PCs. These require low loss transmission through the bulk of the phonic crystal across hundreds or even thousands of lattice periods. In these applications light must couple efficiently from an input slab or rib waveguide to dispersive Bloch modes of the PC at wavelengths outside the PBG (Fig. 1). In this case the entire modal power intersects the porous region and so is potentially subject to scattering loss. In addition, there may be a substantial difference in effective mode index at the interface between the PC and the input rib waveguide leading to further loss due to mode mismatch [7]. Loss reduction and optimisation of coupling to these devices is, therefore, much more challenging.

Loss in PC slab waveguide devices arises from several affects. Firstly, imperfections in fabrication give rise to roughness of the walls of the holes, causing scattering of the waveguide mode into the substrate and surroundings. Secondly, refraction occurs at the boundaries of these structured elements causing light to diverge strongly in the vertical direction. Light propagating across a hole experiences no confinement in the vertical direction (as there are no further dielectric boundaries) and so a small percentage of the modal energy escapes as radiation loss. As the slab waveguide is usually non-symmetric in refractive index profile, most loss occurs due to scattering into the substrate 




Fig. 1. Cross-sectional view of photonic crystal slab waveguide devices showing primary loss mechanisms.

[8]. Divergence within the hole regions was first acknowledged by Krauss et al. [9], who suggested that minimising hole width would help to reduce vertical scattering loss. This principle has been widely adopted for the demonstration of waveguiding PC structures.

Our solution to this problem is rather different [10,11]. By introducing a third material into the holes such that $n_{\text {core }}>n_{\text {rods }} \leftarrow n_{\text {cladding }} \& n_{\text {buffer }}$, greater confinement can be provided for the E-field of an optical signal passing through the guide. Light localised within the holes then experiences a dielectric boundary in the vertical direction which under suitable geometrical conditions enables waveguiding to be maintained within the regions of the holes, reducing the tendency for light to leak into the buffer layer, and divergence at the boundaries of the structured elements.

\section{Experiment 1}

To confirm this theory, a PC structure with holes etched through the cladding and core layer was fabricated. The slab waveguide consisted of a silicon substrate with a $2.2 \mu \mathrm{m}$ thick $\mathrm{SiO}_{2}$ buffer layer formed by thermal oxidation, a $180 \mathrm{~nm}$ thick SiN core layer deposited by LPCVD and a $600 \mathrm{~nm}$ thick $\mathrm{SiO}_{2}$ cladding layer deposited by PECVD. This was then patterned by direct write e-beam lithography and etched by RIE to form an array of air voids.

Broadband transmission spectra were acquired for the two principal symmetry directions on a device with 60 rows of holes arranged in a triangular lattice by end-fire coupling to cleaved facets, using a laser setup previously described [ref]. A drop of calibrated refractive index matching fluid $(n=1.4)$ was then deposited onto the porous region, and the device area sealed with a cover slip (Fig. 2) to prevent evaporation. Spectra were then re-acquired (Fig. 3). All spectra were normalised to the transmission of an unpatterned region of slab waveguide located directly adjacent to the patterned area (Figs. 4 and 5).

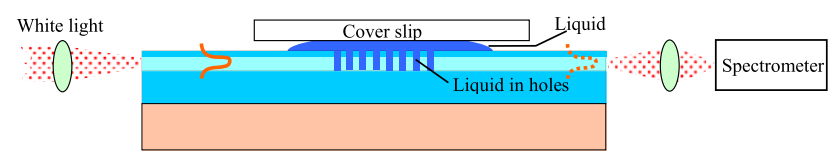

Fig. 2. Cross-sectional view of experimental arrangement for 'liquid filled'.

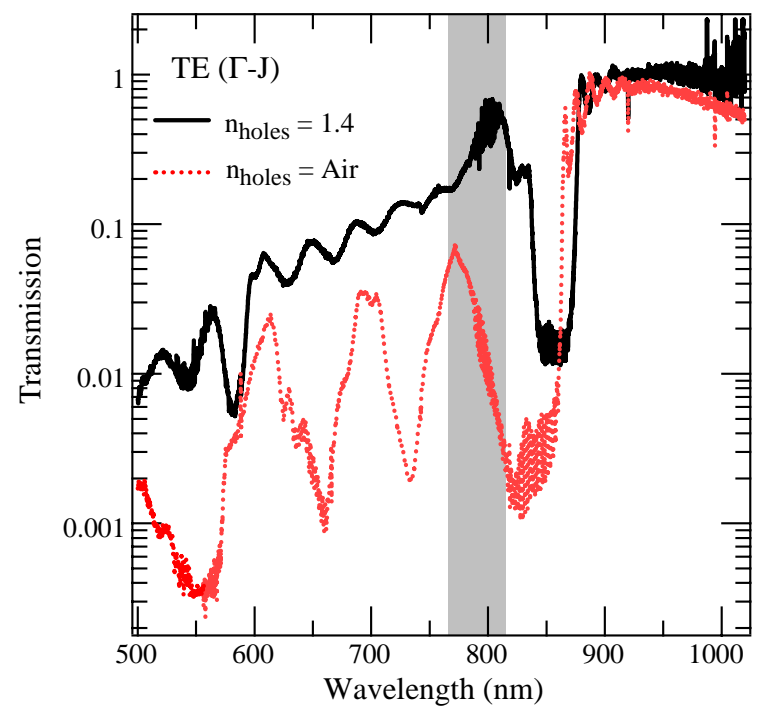

Fig. 3. Transmission spectra ( $T-J$ symmetry direction) comparing air and $n=1.4$ fluid filled holes. Shaded area highlights region of high loss at the lower band edge.

Spectra for the $\Gamma-J$ direction show near unity transmission at wavelengths above the upper band edge tailing off slightly towards longer wavelengths, with little difference between traces for filled/unfilled holes. Below the band-gap spectra for filled/unfilled holes both follow a classic scatering curve with a clear offset in amplitude between the traces.

Primary band gaps are observed for the $\Gamma-X$ direction over $\lambda=811-988 \mathrm{~nm}$ with corresponding extinction ratios of $14.6 \mathrm{~dB}(n=1.4)$ and $18.6 \mathrm{~dB}(n=1)$, and for the $\Gamma-J$ direction over the range $\lambda=771-879 \mathrm{~nm}$ with extinction ratios of $17.5 \mathrm{~dB}(n=1.4), 26 \mathrm{~dB}(n=1)$.

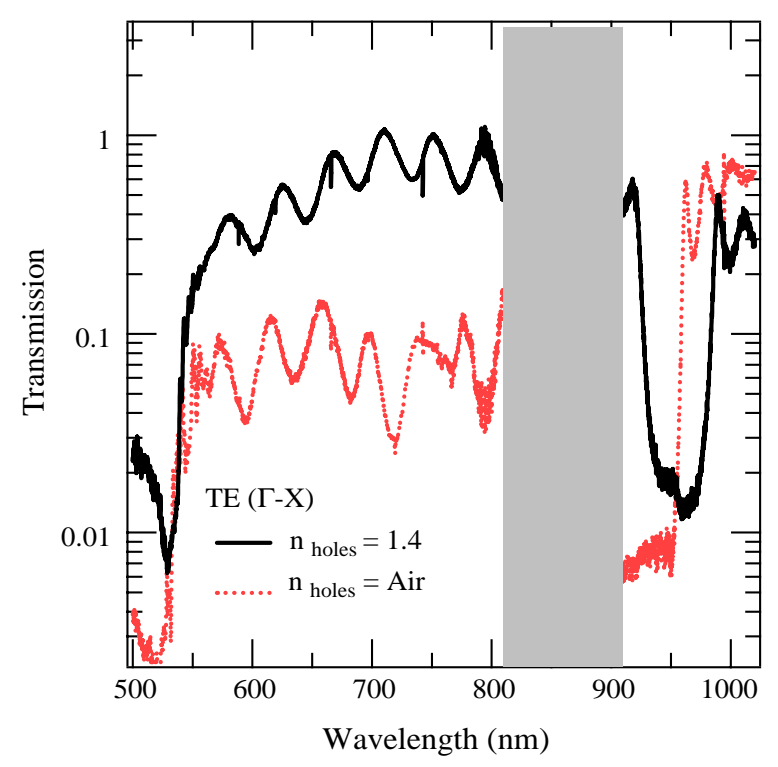

Fig. 4. Transmission spectra ( $T-X$ symmetry direction) comparing air and $n=1.4$ fluid filled holes. Shaded area highlights region of high loss at the lower band edge. 


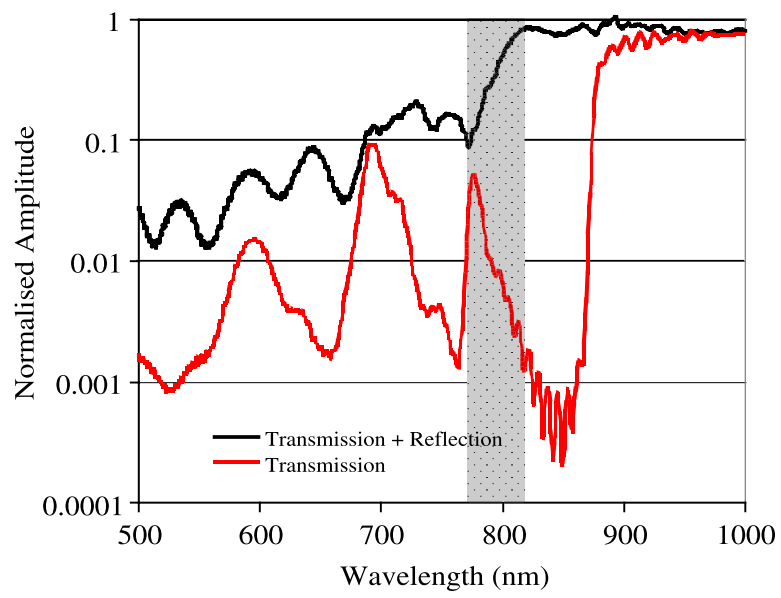

Fig. 5. Theoretical transmission spectra for air filled device ( $I-J$ direction). Shading indicated region of high loss at lower band-edge. Deviation from unity for black trace indicates loss.

Periodic ripples on the spectra are attributed to interference (beating) between waves partially reflected from the boundaries of the patterned region, confirming an effective modeindex mismatch between the input and output slab waveguide regions and the patterned $\mathrm{PC}$ slab waveguide region.

The amplitude offset between filled/unfilled spectra indicates a reduction in loss. This was quantified by measuring the average amplitude offset between the fabry-perot fringes over $\lambda=550-800 \mathrm{~nm}$. Loss was found to be reduced by an average of $4.3 \mathrm{~dB}$ for the $T-X$ direction, and $9.6 \mathrm{~dB}$ for the $\Gamma-J$ direction over this wavelength range. At wavelengths close to the lower band edge (shaded region of graphs) loss was reduced by $19 \mathrm{~dB}$ for both symmetry directions.

At first sight the apparent reduction in loss observed at the lower band-edge could be attributed to an expected reduction in bandgap width due to the reduced refractive index contrast between the holes and the waveguide core material.

To investigate this effect more thoroughly, crosssectional profiles of the patterned slab waveguide structures were modeled using finite difference time domain analysis. In the simulation, both transmission and reflection spectra were acquired simultaneously. An 'ideal' PC device should reflect all light within the wavelength range of the PBG perfectly, whereas all wavelengths outside the bandgaps should be transmitted perfectly, hence by summing transmission and reflection together, scattering and insertion loss can be visualised as a function of wavelength.

Fig. 6 shows the theoretical transmission spectra for unfilled holes. As was the case in the experiment, this was normalised to an un-patterned slab waveguide with identical cross-sectional profile and length hence effects due to intrinsic material absorption and waveguide dispersion have effectively been removed from the plot.

As was the case for the experiment, near unity transmission is observed at wavelengths above the upper band edge and some distance into the band gap. At wavelengths closer to the lower band-edge (shaded region of the plot) loss increases dramatically, confirming the fact that light which should couple from the input waveguide to the PC device at the lower band edge, actually scatters out of the device, causing 'rounding' of the lower band edge.

It now becomes clear that the experimental data shown in Figs 4 and 5 show greatly reduced loss and enhanced coupling for modes lying close to the lower band gap edge. This result has extremely important implications for applications which make use of the dispersive properties of these modes such as super-prisms and optical time delay elements.

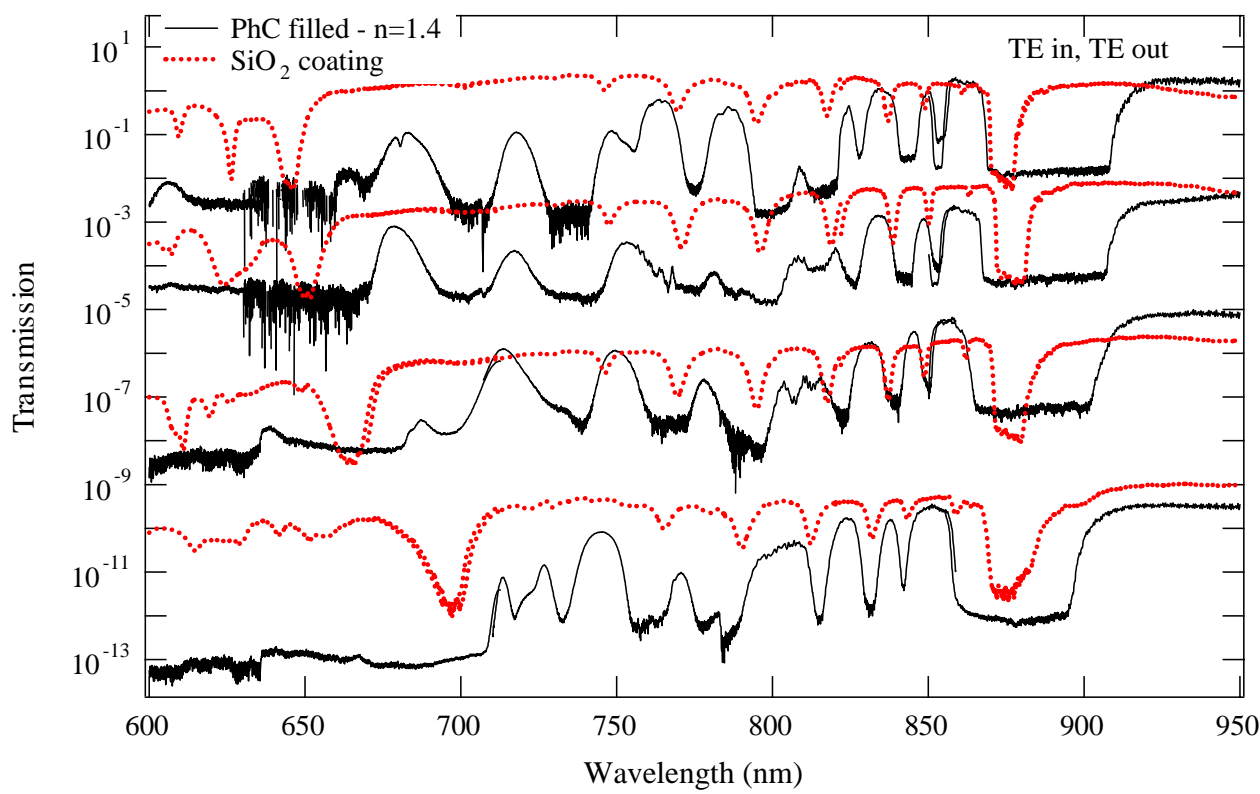

Fig. 6. Transmission spectra for liquid filled holes and $\mathrm{SiO}_{2}$ overcoated samples. 


\section{Experiment 2}

Although the optimal geometric arrangement would be for $n_{\text {rods }}>n_{\text {cladding, }}$, the added complexity of fabrication of such structures incorporating a third dielectric material may in practice make their implementation impractical or very costly. An alternative solution is to fill the holes with the cladding material. This improves confinement somewhat by reducing divergence, and provides vertical confinement in the regions of the holes. However, leakage to substrate modes may still occur.

To investigate this further, slab waveguides similar to those used in the 'liquid filled holes experiment' were grown, but this time arrays of holes were patterned and etched directly into the core layer. The resultant porous waveguide core was then overcoated with a $600 \mathrm{~nm}$ thick $\mathrm{SiO}_{2}$ cladding layer by PECVD.

An experimental comparison was made between a device with liquid-filled holes $(n=1.4)$ and a device overcoated with $\mathrm{SiO}_{2}$ by PECVD. Spectra for devices consisting of 600 rows of holes arranged on a rectangular lattice with an aspect ratio of 1:1.5 and a pitch of $310 \mathrm{~nm}$ (measured along the minor axis of the rectangle), and nominal hole diameter of $180 \mathrm{~nm}$, are shown in Fig. 6 . Four different directions of propagation were investigated. Traces for each direction were separated on the graph for clarity of comparison.

Black traces show spectra for a liquid filled device. Both edges of the primary band-gaps are extremely well defined with an extinction ratio better than $20 \mathrm{~dB}$ in the band-gap region. In addition, there are several well defined dips in transmission corresponding to dispersive features of the photonic band-structure. The position of these features shift only slightly with direction of propagation. Overall transmission reduces significantly as wavelength gets smaller, and at wavelengths below $675 \mathrm{~nm}$ the liquid filled spectra become extremely lossy.

Red traces show spectra for $\mathrm{SiO}_{2}$ over-coated samples. Again the edges of the primary band gap are very well defined, but its width is somewhat reduced due to a reduction in $\Delta n$ between the holes and the core material. As was the case for the liquid filled samples, dispersive dips are observed at wavelengths below the lower band edge, in roughly the same positions, however, they are now more sharply defined and transmission returns to near unity in between.

The most significant feature of the $\mathrm{SiO}_{2}$ over-coated spectra, is the fact that the overall transmission amplitude remains near unity over the entire wavelength range of the scan. In fact the loss for the over-coated sample was found to increase by just $0.07 \mathrm{~dB}$ across the entire wavelength range $680-900 \mathrm{~nm}$. Strong dips are also observed over the wavelength range $600-700 \mathrm{~nm}$. These change position rapidly with direction of propagation, and correspond to higher order band-gaps of the PC. These features are not apparent at all in the liquid filled spectra due to high losses at those short wavelengths.
The differential in loss between the liquid filled and $\mathrm{SiO}_{2}$ overcoated sample was again quantified by comparing the amplitude of several peeks across the wavelength range $600-850 \mathrm{~nm}$. Loss was found to be reduced by an average of $16 \mathrm{~dB}$ across this entire wavelength range, varying from $10 \mathrm{~dB}$ at $785 \mathrm{~nm}$ to $20 \mathrm{~dB}$ at $600 \mathrm{~nm}$.

Overall insertion loss (excluding facet coupling loss) for the overcoated PC device was measured to be just $4.5 \mathrm{~dB}$. The majority of this insertion loss can be attributed to a missmatch in effective mode index between the input and output slab waveguide regions and the PC region, hence, loss does not in this case scale significantly with number of rows.

This result has extremely important implications for the realisation of commercially viable super-prism and time delay components, whose performance is largely limited by a trade off between total number of rows of lattice periods and insertion loss. Furthermore, as these applications do not necessarily require the existence of a PBG but merely require the existence of strongly dispersive features, our novel loss reduction mechanism allows higher order dispersion bands to be utilised for these applications. This greatly reduces fabrication tolerance since required lattice pitch scales directly with dispersion band number.

\section{Conclusions}

In conclusion, we have demonstrated a significant milestone in the reduction of scattering loss in PC slab waveguide devices. Loss was reduced by $4-9 \mathrm{~dB}$ over the spectral range $500-1000 \mathrm{~nm}$ by incorporating index matched fluid in the holes of an otherwise lossy PC device. Loss was reduced by a further $10-20 \mathrm{~dB}$ over the same wavelength range by filling the holes with $\mathrm{SiO}_{2}$ using a more rigorous microfabrication process. Insertion loss of the resultant PC region was measured to be just $4.5 \mathrm{~dB}$, most of which was shown to be attributed to coupling loss. Loss was found to increase by just $0.07 \mathrm{~dB}$ over the entire visible spectra. This is to our knowledge the first detailed practical or theoretical investigation of broadband loss in PC slab waveguides, and the first practical realisation of a PC device capable of using higher order PBGs in applications such as super-prisms and optical time delay elements.

\section{References}

[1] J.D. Joannopoulos, P.R. Villeneuve, S. Fan, Photonic crystals putting a new twist on light, Nature 386 (1997) 143-149.

[2] A. Yariv, Y. Xu, R.K. Lee, A. Scherer, Coupled-resonator optical waveguide: a proposal and analysis, Opt. Lett. 24 (11) (1999) 711-713.

[3] H. Kosaka, T. Kawashima, A. Tomita, M. Notomi, T. Tamamura, T. Sato, S. Kawakam, Superprism phenomena in photnic crystals: toward microscale lightwave circuits, J. Lightwave. Technol. 17 (11) (1999) 2032. 
[4] J.J. Baumberg, N.M.B. Perney, M.C. Netti, M.D.B. Charlton, M.E. Zoorob, G.J. Parker, Visible-wavelength super-refraction in photonic crystal superprisms, Appl. Phys. Lett. 85 (3) (2004) 354 356.

[5] S.N.H. Zheng, H. Chen, Time delay of light propagation through defect modes of one-dimensional photonic band-gap structures, Opt. Commun. 174 (1-4) (2000) 139-144.

[6] M.C. Netti, C.E. Finlayson, J.J. Baumberg, M.D.B. Charlton, M.E. Zoorob, J.S. Wilkinson, G.J. Parker, Separation of photonic crystal waveguides modes using femtosecond time-of-flight, Appl. Phys. Lett. 81 (21) (2002) 3927-3929.
[7] M.D.B. Charlton, S.W. Roberts, G.J. Parker, Mater. Sci. Eng. B 49 (1997) 155 .

[8] R. Ferrini, R. Houdre, H. Benistry, M. Qiu, J. Moosburger, Radiation losses in planar photonic crystals: two-dimensional representation of hole depth and shape by an imaginary dielectric constant, J. Opt. Soc. Am. B: Opt. Phys. 20 (3) (2003) 469-478.

[9] T.F. Krauss, R.M. De La Rue, S. Brand, Nature 383 (1996) 699.

[10] G.J. Parker, M.D.B. Charlton, M.E. Zoorob, US Patent, application no. 20040146257

[11] G.J. Parker, M.D.B. Charlton, M.E. Zoorob, US Patent, application no. 20040067035. 